KEK-TH-1881, J-PARC-TH-0047

\title{
Constituent-counting rule in photoproduction of hyperon resonances
}

\author{
Wen-Chen Chang, ${ }^{1}$ S. Kumano, ${ }^{2,3}$ and Takayasu Sekihara ${ }^{4}$ \\ ${ }^{1}$ Institute of Physics, Academia Sinica, Taipei 11529, Taiwan \\ ${ }^{2}$ KEK Theory Center, Institute of Particle and Nuclear Studies, \\ High Energy Accelerator Research Organization (KEK), \\ 1-1, Ooho, Tsukuba, Ibaraki, 305-0801, Japan \\ ${ }^{3}$ J-PARC Branch, KEK Theory Center, Institute of Particle and Nuclear Studies, KEK, \\ and Theory Group, Particle and Nuclear Physics Division, J-PARC Center, \\ 203-1, Shirakata, Tokai, Ibaraki, 319-1106, Japan \\ ${ }^{4}$ Research Center for Nuclear Physics (RCNP), Osaka University, Ibaraki, Osaka 567-0047, Japan
}

(Dated: March 2, 2022)

\begin{abstract}
We analyze the CLAS data on the photoproduction of hyperon resonances, as well as the available data for the ground state $\Lambda$ and $\Sigma^{0}$ of the CLAS and SLAC-E84 collaborations, by considering constituent-counting rule suggested by perturbative QCD. The counting rule emerges as a scaling behavior of cross sections in hard exclusive reactions with large scattering angles, and it enables us to determine the number of elementary constituents inside hadrons. Therefore, it could be used as a new method for identifying internal constituents of exotic-hadron candidates. From the analyses of the $\gamma p \rightarrow K^{+} \Lambda$ and $K^{+} \Sigma^{0}$ reactions, we find that the number of the elementary constituents is consistent with $n_{\gamma}=1, n_{p}=3, n_{K^{+}}=2$, and $n_{\Lambda}=n_{\Sigma^{0}}=3$. Then, the analysis is made for the photoproductions of the hyperon resonances $\Lambda(1405), \Sigma(1385)^{0}$, and $\Lambda(1520)$, where $\Lambda(1405)$ is considered to be a $\bar{K} N$ molecule and hence its constituent number could be five. However, we find that the current data are not enough to conclude the numbers of their constituent. It is necessary to investigate the higher-energy region at $\sqrt{s}>2.8 \mathrm{GeV}$ experimentally beyond the energy of the available CLAS data for counting the number of constituents clearly. We also mention that our results indicate energy dependence in the constituent number, especially for $\Lambda(1405)$. If an excited hyperon is a mixture of three-quark and five-quark states, the energy dependence of the scaling behavior could be valuable for finding its composition and mixture.
\end{abstract}

PACS numbers: 12.38.Bx, 13.60.Rj, 14.20.Pt

\section{INTRODUCTION}

Almost all the hadrons are described by assuming that they are composed of minimal numbers of quarks to construct color singlet states, i.e., three quarks ( $q q q)$ for baryons and a quarkantiquark pair $(q \bar{q})$ for mesons, in conventional quark models [1]. However, the fundamental theory of strong interaction, quantum chromodynamics (QCD), allows us to consider hadrons whose structure cannot be classified into $q q q$ nor $q \bar{q}$, such as pentaquark systems $(q q q q \bar{q})$, as long as they are color singlet states. They are called "exotic" hadrons. One of the exotic hadron candidates is the lowest hyperon resonance, $\Lambda(1405)$, which has been expected to be a $\bar{K} N$ bound state rather than a $u d s$ state for a long time [2]. In addition, it is very encouraging that recent experimental analyses in heavyquark sector discovered charged quarkonium-like states [3] and charmonium-pentaquark states [4] as candidates of the exotic hadrons.

In order to conclude the structure of an exotic hadron candidate, it is essential to clarify quark-gluon composition of the hadron. For example, the total number of constituent quarks and antiquarks is $n(q)+n(\bar{q})=3$ for a usual $q q q$ baryon, but it becomes $n(q)+n(\bar{q})=5$ for a pentaquark $q q q q \bar{q}$ state. Although one cannot, in general, count the total number of quarks and antiquarks inside a hadron due to the annihilation and creation of a quark-antiquark pair, there is an exception in hard exclusive reactions with large scattering angles. There, the so-called constituent-counting rule emerges as a scaling behavior of the cross sections according to per- turbative QCD [5, 6]. Actually, in the constituent-counting rule, the scaling factor for the cross section corresponds to the total number of elementary constituents involved in the hardexclusive reaction. This enables us to determine the number of elementary constituents inside hadrons.

In the experimental side, the constituent-counting rule was first applied in Ref. [7] to the reactions $\gamma p \rightarrow \pi^{+} n$, $\pi^{0} p, \pi^{-} \Delta^{++}, \rho^{0} p, K^{+} \Lambda$, and $K^{+} \Sigma^{0}$ by using the photoproduction data of the SLAC (SLAC National Accelerator Laboratory)-E84 experiment, implying the number of the constituent $n=3$ for the baryons and $n=2$ for the mesons. The $\gamma p \rightarrow \pi^{+} n$ reaction was analyzed recently by the JLab (Thomas Jefferson National Accelerator Facility) Hall-A and E94-104 collaborations [8, 9], and they found that the experimental data are consistent with $n_{\gamma}=1, n_{p}=n_{n}=3$, and $n_{\pi^{+}}=2$. Recent data on the $\Lambda$ photoproduction from CLAS at JLab were analyzed in Ref. [10], and the expected $n=9$ scaling appears to be valid for $\gamma p \rightarrow K^{+} \Lambda$. Various meson photoproductions from CLAS were investigated in Ref. [11], but the author could not conclude the scaling for the vector mesons as suggested in conventional quark models due to additional gluon exchanges from higher Fock states for hadrons. The constituent-counting rule was also confirmed by two-body hadronic exclusive reactions at BNL (Brookhaven National Laboratory)-AGS (Alternating Gradient Synchrotron) [12]. It is noteworthy that the constituentcounting rule was experimentally shown to be valid even for nuclei [13, 14]; the numbers of elementary constituents inside the deuteron, ${ }^{3} \mathrm{H}$, and ${ }^{3} \mathrm{He}$ are counted as $n_{d}=6$ and $n_{3^{3}}=n_{3} \mathrm{He}=9$. 
On the other hand, there are theoretical proposals recently to use the constituent-counting rule for clarifying the internal structure of hadrons, especially exotic-hadron candidates 15 19]. Actually, if a baryon (meson) contains more than three (two) constituents as an exotic hadron, it should explicitly affect the scaling behavior of its production cross section at high energies. Moreover, since the number of elementary constituents inside nuclei is counted as $n_{d}=6$ and $n_{3^{\mathrm{H}}}=n_{3} \mathrm{He}=9[13,14]$, we can apply the constituentcounting rule not only to compact exotic hadrons but also to hadronic molecules composed of two (or more) color singlet states, although we cannot distinguish whether the hadron is a compact multiquark system or a hadronic molecule solely by the constituent-counting rule. For instance, the number of constituents inside $\Lambda(1405)$ is considered to be five if it is a $\bar{K} N$ bound state, which cannot be distinguished from a compact pentaquark state only by the scaling behavior. Nevertheless, there are possibilities that compact multiquark and diffuse hadron-molecule states could be further distinguished by the so-called compositeness [20-23] and hadron tomography in terms of three-dimensional structure functions, generalized parton distributions (GPDs), transverse-momentumdependent parton distributions (TMDs), and generalized distribution amplitudes (GDAs) [16]. However, much efforts should be made for such tomography studies because there is no experimental information on these observables for exotic hadron candidates at this stage.

In this article, we use the constituent-counting rule as a guiding principle in analyzing available experimental data on hard exclusive cross sections at high energies. Particularly, we investigate new data obtained by the CLAS collaboration at JLab for the photoproduction of the hyperon resonances $\Lambda(1405), \Sigma(1385)^{0}$, and $\Lambda(1520)$ from the proton target $\left(\gamma p \rightarrow K^{+} Y^{(*)}\right)$ [24], extending the preceding studies on the scaling behavior for the ground-state hyperons. Among the hyperon resonances, $\Lambda(1405)$ is expected to be a $\bar{K} N$ molecule [2] and hence its constituent number could be five, so that it is interesting to obtain the constituent numbers of these exotic hadron candidates. The hyperons $\Sigma(1385)^{0}$ and $\Lambda(1520)$ are commonly considered as ordinary baryons with the $q q q$ configuration; however, there are some theoretical studies that $\Lambda(1520)$ may have a certain fraction of hadronic molecular components [25]. We also analyze new CLAS data for $\Lambda$ and $\Sigma^{0}$ [26, 27] and the SLAC-E84 data [7]. In the CLAS experiments, the photon momentum in the laboratory frame is up to $3.8 \mathrm{GeV} / c$ and hence the center-of-mass energy is up to $2.8 \mathrm{GeV}$. We check the scaling behavior of the photoproduction cross sections and investigate a possibility to count the elementary constituents inside the hadrons.

This article is organized in the following way. In Sec. II, the constituent-counting rule and the cross section is briefly explained. The photoproduction cross-section data are analyzed, and cross-section slopes $1 / s^{n-2}$ are examined at high energies to show the constituent numbers $n$ in Sec. III We summarize our studies in Sec. IV]

\section{CONSTITUENT-COUNTING RULE IN HARD EXCLUSIVE REACTIONS}

We introduce the constituent counting rule for a hard exclusive reaction by showing expressions for its cross section and matrix element and explaining a typical hard-gluon-exchange process which contributes to the exclusive reaction. The cross section for an exclusive reaction $a+b \rightarrow c+d$ is given by the matrix element $M_{a b \rightarrow c d}$ as

$$
\frac{d \sigma_{a b \rightarrow c d}}{d t} \simeq \frac{1}{16 \pi s^{2}} \sum_{\text {pol }}\left|M_{a b \rightarrow c d}\right|^{2} .
$$

Here, the approximation means that the masses of particles are neglected, and the Mandelstam variables $s$ and $t$ are defined by the momenta of the particles, $p_{i}(i=a, b, c, d)$, as

$$
\begin{aligned}
& s=\left(p_{a}+p_{b}\right)^{2} \simeq 4\left|\vec{p}_{\mathrm{cm}}\right|^{2} \\
& t=\left(p_{a}-p_{c}\right)^{2} \simeq-2\left|\vec{p}_{\mathrm{cm}}\right|^{2}\left(1-\cos \theta_{\mathrm{cm}}\right),
\end{aligned}
$$

where $p_{\mathrm{cm}}$ and $\theta_{\mathrm{cm}}$ are momentum and scattering angle in the c.m. frame, respectively. The average over the initial spins and the summation for the final spins are taken in Eq. (1).

In this article, we investigate hard photoproductions of hadrons, so that the reaction $\gamma+b \rightarrow c+d$ is considered in the following discussions. The matrix element is denoted in general by the photon-parton scattering amplitude $H_{\gamma b \rightarrow c d}$ and the light-cone distribution amplitudes of hadrons, $\phi_{b}, \phi_{c}$, and $\phi_{d}$, as [28]

$$
\begin{aligned}
M_{\gamma b \rightarrow c d}= & \int\left[d x_{b}\right]\left[d x_{c}\right]\left[d x_{d}\right] \phi_{c}\left(\left[x_{c}\right]\right) \phi_{d}\left(\left[x_{d}\right]\right) \\
& \times H_{\gamma b \rightarrow c d}\left(\left[x_{b}\right],\left[x_{c}\right],\left[x_{d}\right], Q^{2}\right) \phi_{b}\left(\left[x_{b}\right]\right),
\end{aligned}
$$

as shown in Fig. 11. Here, $[x]$ indicates a set of the light-cone momentum fractions of partons in a hadron, $x_{j}=p_{j}^{+} / p^{+}$ with $j$ th parton and hadron momenta $p_{j}$ and $p$, respectively. The light-cone component is defined as $p^{+}=\left(p^{0}+p^{3}\right) / \sqrt{2}$ with the choice of the third axis for the longitudinal direction. The hadron distribution amplitude $\phi_{i}([x])$ is the amplitude for finding quarks with the momentum fractions $[x]$ in the hadron $i$.

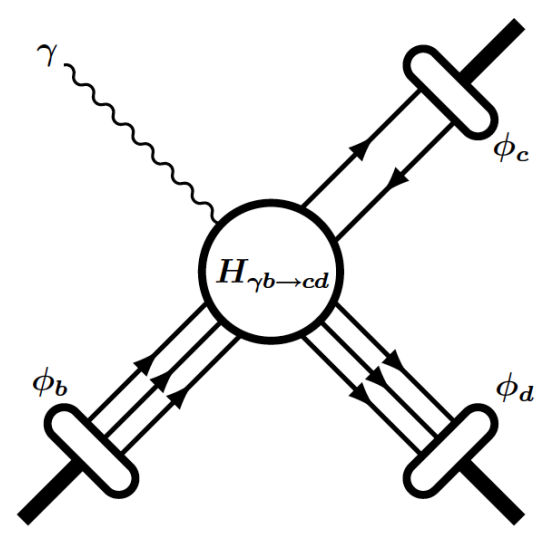

FIG. 1: Hard exclusive reaction $\gamma+b \rightarrow c+d$. 
A hard exclusive process $\gamma+b \rightarrow c+d$ can occur if a large momentum transfer is shared by exchanged hard gluons as shown in Fig. 2. Considering hard gluon and quark propagators in the intermediate state together with normalization factors of initial and final quarks, we have the scaling property for its cross section at high-momentum transfer in terms of the number of constituents which participate in the reaction [15]. Its cross section is expressed as [5, 6]

$$
\frac{d \sigma}{d t}=\frac{1}{s^{n-2}} f(t / s),
$$

by the Mandelstam variables $s$ and $t$. Here, $f(t / s)$ is a function of a variable $t / s$ which corresponds to the center-of-mass scattering angle $\theta_{\mathrm{cm}}$ in the high-energy region [see Eq. (2)]. The scaling factor $n$ counts the number of the participating constituents. In the $\gamma+b \rightarrow c+d$ reaction, we have $n=n_{\gamma}+n_{b}+n_{c}+n_{d}$, where the particle $i(=\gamma, b, c$, and $d$ ) is made of $n_{i}$ elementary constituents. Therefore, from the scaling law of the cross section (4), we can learn the total number of constituents $n$. The detailed formulation of the constituent-counting rule is found in Ref. [15].

Although the constituent-counting rule is a useful guideline, we do not know the energy at which the scaling starts. If the absolute cross section were calculated precisely from the low-energy resonance region to the high-energy perturbative QCD region, the transition point should be determined. The resonance region could be theoretically described relatively well by effective hadron models. However, it is not the case in the high-energy region, although the high-energy slope should be determined by the constituent counting rule in Eq. (4).

There are two major difficulties in calculating the absolute cross section in Eq. (1). The hadron distribution amplitudes $\phi_{i}([x])$ are not well investigated except for the pion [29], so that the amplitude $H_{\gamma b \rightarrow c d}\left(\left[x_{b}\right],\left[x_{c}\right],\left[x_{d}\right], Q^{2}\right)$ cannot be calculated reliably. Second, there are many diagrams which contribute to the exclusive reaction depending how the gluons couple to quarks. For instance, we show a typical diagram for the process $\gamma p \rightarrow \pi^{+} n$ in Fig. 2] which does not look complicated. However, as the quark numbers grow especially in exotic hadrons, the number of diagrams increases significantly, so that development of an automatic code to obtain Feynman diagrams and to estimate their numerical contributions is essential. Unless we complete these serious studies, it is theoretically impossible to calculate the absolute magnitude

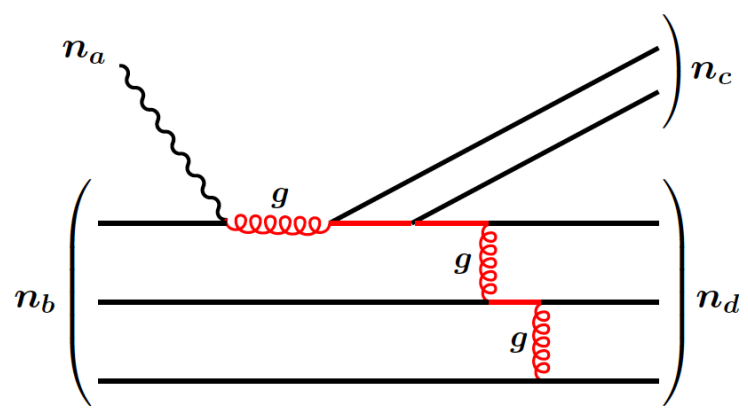

FIG. 2: (color online) A typical hard-gluon-exchange process in the exclusive reaction $\gamma+b \rightarrow c+d$ at high energies. of an exclusive cross section, hence to obtain the minimum energy for the scaling.

In spite of these issues for estimating the absolute cross section, the constituent counting rule itself is considered to be valid by perturbative QCD for a hard exclusive reaction. We use this scaling rule for finding the numbers of elementary constituents which participate in the reaction. This should be used for exotic hadron studies because the number of constituent quarks should be different from ordinary hadrons $(q \bar{q}$, $q q q)$.

\section{ANALYSIS RESULTS}

In this study we consider the $\gamma p \rightarrow K^{+} Y^{(*)}$ reactions with $Y^{(*)}$ being the $\Lambda, \Sigma^{0}, \Lambda(1405), \Sigma(1385)^{0}$, and $\Lambda(1520)$ hyperons. The reactions $\gamma p \rightarrow K^{+} \Lambda$ and $K^{+} \Sigma^{0}$ are observed in SLAC-E84 [7] and CLAS [26, 27], and the reactions $\gamma p \rightarrow K^{+} \Lambda(1405), K^{+} \Sigma(1385)^{0}$, and $K^{+} \Lambda(1520)$ in CLAS [24]. In the CLAS experiment, they measured the differential cross sections $d \sigma / d \cos \theta_{\mathrm{cm}}$. This can be translated into the differential cross section $d \sigma / d t$ by the formula

$$
\frac{d \sigma}{d t}=\frac{1}{2 p_{\text {in }} p_{\text {out }}} \frac{d \sigma}{d \cos \theta_{\mathrm{cm}}},
$$

where $p_{\text {in }}\left(p_{\text {out }}\right)$ is the center-of-mass momenta of the particles in the initial (final) state. Since the scaling behavior appears in the large scattering-angle region, we take the data in scattering-angle bins around the right angle $\theta_{\mathrm{cm}}=90^{\circ}$ in the following analyses. Here we note that, as explained in the previous section, we do not know beforehand the minimum energy for the scaling, so that in the present analyses we examine several values of the minimal energy for the scaling, $\sqrt{s_{\min }}$, on a case-by-case basis for each reaction. We did not analyze the $\gamma n \rightarrow K^{+} \Sigma^{-}$cross section from CLAS [30] because for its measurement they used bound neutron in the deuteron, for which we may need to take into account nuclear effects.

The analyses are done in the following way. We assume that the differential cross section $d \sigma / d t$ is given by the form $f\left(\theta_{\mathrm{cm}}\right) / s^{n-2}$ with the scattering-angle-dependent function $f\left(\theta_{\mathrm{cm}}\right)$. The function $f\left(\theta_{\mathrm{cm}}\right)$ takes different values for different bins:

$$
f\left(\theta_{\mathrm{cm}}\right)_{(1)}, f\left(\theta_{\mathrm{cm}}\right)_{(2)}, \cdots, f\left(\theta_{\mathrm{cm}}\right)_{(N)},
$$

in fitting $N$ bins of the scattering angle. Here, the constants $f\left(\theta_{\mathrm{cm}}\right)_{(1)}, \cdots, f\left(\theta_{\mathrm{cm}}\right)_{(N)}$ are also fitting parameters. The scaling factor $n$ is fixed regardless of the scattering angle. Namely, the differential cross section $d \sigma / d t$ is fitted by the function $f\left(\theta_{\mathrm{cm}}\right) / s^{n-2}$ with $N+1$ parameters, $f\left(\theta_{\mathrm{cm}}\right)_{(1)}$, $\cdots, f\left(\theta_{\mathrm{cm}}\right)_{(N)}$, and $n$. In the fitting procedure, we mean 5 bins for the bins from $-0.25<\cos \theta_{\mathrm{cm}}<-0.15$ to $0.15<\cos \theta_{\text {cm }}<0.25$ with 0.10 step each; 4 bins from $-0.2<\cos \theta_{\mathrm{cm}}<-0.1$ to $0.1<\cos \theta_{\mathrm{cm}}<0.2 ; 2$ bins from $-0.1<\cos \theta_{\mathrm{cm}}<0.0$ to $0.0<\cos \theta_{\mathrm{cm}}<0.1$; and 1 bin for $-0.05<\cos \theta_{\mathrm{cm}}<0.05$. The results are shown in Figs. 3 4 5, 6, 7, 8, and 9, and Tables \and 


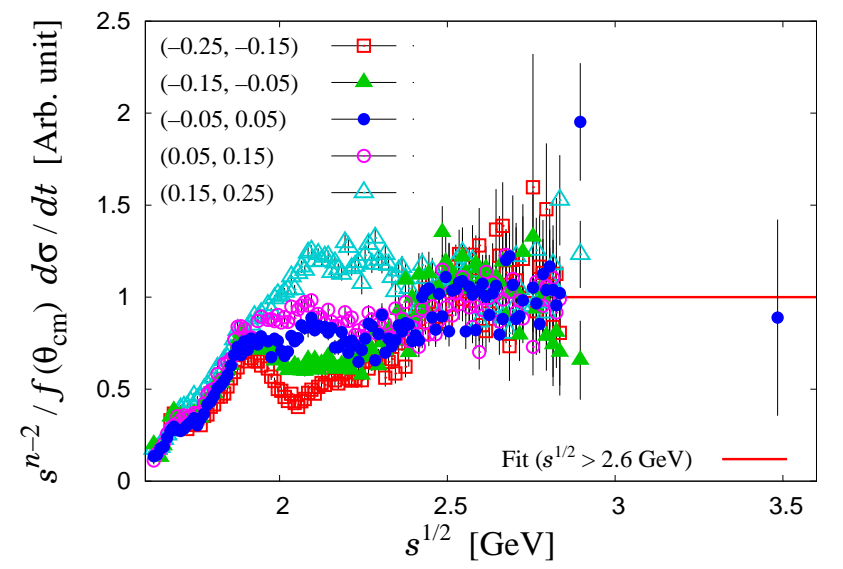

FIG. 3: (color online) Experimental data of $\gamma p \rightarrow K^{+} \Lambda$ cross section $d \sigma / d t$ [7, 26] multiplied by $s^{n-2} / f\left(\theta_{\mathrm{cm}}\right)(n=10.0)$ with the function $f\left(\theta_{\mathrm{cm}}\right)$ in Eq. (6). The scaling factor $n$ is fixed at 10.0 by fitting in the region $\sqrt{s} \geq 2.6 \mathrm{GeV}$ as shown in Table 1 The solid line indicates a fit to the data.

First, there are many data on the photoproductions of $\Lambda$ and $\Sigma^{0}$ as shown in Figs. 3 and 4 . There are five sets of data in each figure. For the $\Lambda$ production in Fig. 3, the scaling is approximately seen for the bin of $-0.05<\cos \theta_{\mathrm{cm}}<0.05$, by including the high-energy data at $3.5 \mathrm{GeV}$ from the SLACE84 experiment. Other bin data deviate slightly form the scaling behavior of $n \simeq 10$ suggested by the $-0.05<\cos \theta_{\mathrm{cm}}<$ 0.05 bin data. Obviously, we need measurements to fill the gap in the region, 3.0 $\mathrm{GeV}<\sqrt{s}<3.5 \mathrm{GeV}$ between the existing data points. For the $\Sigma^{0}$ production in Fig. 4, the discrepancies between the data of different bins are more pronounced at low energies $(\sqrt{s}<2.3 \mathrm{GeV})$. However, they seem to merge into the scaling function at $\sqrt{s}>2.6 \mathrm{GeV}$. The scaling is rather clear in the data of the bin $-0.05<$ $\cos \theta_{\mathrm{cm}}<0.05$ if the data at $\sqrt{s}=3.5 \mathrm{GeV}$ is taken into account. However, more data should be obtained in the energy region $3.0 \mathrm{GeV}<\sqrt{s}<3.5 \mathrm{GeV}$ for drawing a clearer conclusion on the scaling behavior in the same way with the $\Lambda$ production in Fig. 3

Next, we determine the scaling factor $n$ from the highenergy data at $s \geq s_{\min }$ by excluding low-energy resonances. As expected from Figs. 3 and 4 the obtained factor $n$ depends on the choice of $s_{\min }$. By using all the five-bin data or the one-bin data for the $\Lambda$ and $\Sigma^{0}$ productions, we determine the factor $n$ and the results are shown in Table $\square$ and Fig. 5 for various choices of $\sqrt{s_{\min }}$ from $2.3 \mathrm{GeV}$ to $2.7 \mathrm{GeV}$. If the onebin $\left(-0.05<\cos \theta_{\mathrm{cm}}<0.05\right)$ data are used, the determined number is consistent with $n=9$, namely with the assignment $n_{\gamma}=1, n_{p}=3, n_{K^{+}}=2, n_{\Lambda}=3$, and $n_{\Sigma^{0}}=3$ although the detailed values depend on $s_{\min }$. Even if the five-bin data are used, the determined values of $n$ are almost same by considering errors except for the ones at $\sqrt{s} \geq \sqrt{s_{\min }}=2.3$ and $2.4 \mathrm{GeV}$ for $\Sigma^{0}$. These results indicate that the constituent numbers determined by the constituent-counting rule are consistent with the standard quark model compositions, $n_{\Lambda}=3$ and $n_{\Sigma^{0}}=3$.

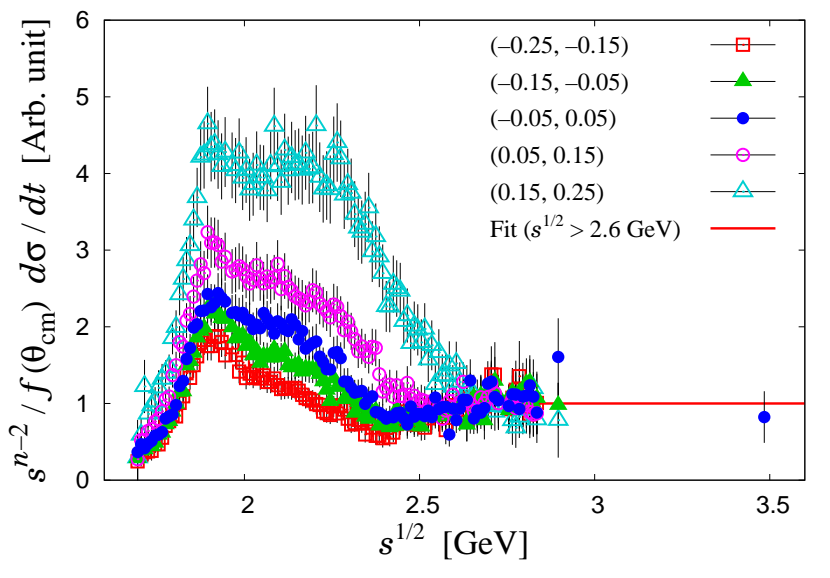

FIG. 4: (color online) Experimental data of $\gamma p \rightarrow K^{+} \Sigma^{0}$ cross section $d \sigma / d t$ [7, 27] multiplied by $s^{n-2} / f\left(\theta_{\mathrm{cm}}\right)(n=9.2)$ with the function $f\left(\theta_{\mathrm{cm}}\right)$ in Eq. (6). The scaling factor $n$ is fixed at 9.2 by fitting in the region $\sqrt{s} \geq 2.6 \mathrm{GeV}$ as shown in Table【 The solid line is a fit to the data.

TABLE I: Determined values of the scaling factor $n$ for $\gamma p \rightarrow K^{+} \Lambda$ and $K^{+} \Sigma^{0}$. We also show the $\chi^{2}$ values divided by the degrees of freedom $N_{\text {d.o.f. }}$.

\begin{tabular}{|c|c|c|}
\hline \multicolumn{3}{|c|}{$\gamma p \rightarrow K^{+} \Lambda$} \\
\hline$\sqrt{s_{\min }}$ & $n\left(\chi^{2} / N_{\text {d.o.f. }}\right), 5$ bins & $n\left(\chi^{2} / N_{\text {d.o.f. }}\right), 1$ bin \\
\hline $2.3 \mathrm{GeV}$ & $9.5 \pm 0.1 \quad(527 / 257)$ & $9.2 \pm 0.1 \quad(71 / 52)$ \\
\hline $2.4 \mathrm{GeV}$ & $(252 / 207)$ & $9.5 \pm 0.2$ \\
\hline $2.5 \mathrm{GeV}$ & $10.2 \pm 0.2$ & $9.7 \pm 0.3$ \\
\hline $2.6 \mathrm{GeV}$ & $10.0 \pm 0.2$ & $9.6 \pm 0.5$ \\
\hline $2.7 \mathrm{GeV}$ & $9.7 \pm 0.6$ & $9.2 \pm 0.7$ \\
\hline & $\gamma p \rightarrow K^{+} \Sigma^{0}$ & \\
\hline$\sqrt{s_{\min }}$ & $n\left(\chi^{2} / N_{\text {d.o.f. }}\right), 5$ bins & $n\left(\chi^{2} / N_{\text {d.o.f. }}\right), 1$ bin \\
\hline $2.3 \mathrm{GeV}$ & $10.5 \pm 0.2 \quad(387 / 257)$ & $9.3 \pm 0.2 \quad(34 / 52)$ \\
\hline $2.4 \mathrm{GeV}$ & $(191 / 207)$ & $8.7 \pm 0.2$ \\
\hline $2.5 \mathrm{GeV}$ & $9.2 \pm 0.2$ & $8.8 \pm 0.3$ \\
\hline $2.6 \mathrm{GeV}$ & $9.2 \pm 0.2$ & $9.1 \pm 0.4$ \\
\hline $2.7 \mathrm{GeV}$ & $10.2 \pm 0.3$ & $9.8 \pm 0.4$ \\
\hline
\end{tabular}

One of the major purposes of this work is to apply the constituent-counting rule to exotic-hadron candidates for determining the numbers of constituents for them. It should be a significant step in the study of exotic hadrons to apply a new approach, which is rather different from the current ones with low-energy observables such as mass and decay width, to clarify their internal structure.

In this study, we analyze the photoproductions of $\Lambda$ (1405), $\Sigma(1385)^{0}$, and $\Lambda(1520)$. Especially, $\Lambda(1405)$ is speculated to be a $\bar{K} N$ bound state or possibly a mixture of $\bar{K} N$ bound state, compact five-quark system, and conventional threequark one, so that its number of constituents might be different from that for an ordinary baryon $\left(n_{\Lambda(1405)} \neq n_{\Lambda}=3\right)$. The results are shown in Figs. 6, 7 and 8 As obvious from these figures, there are not enough data, and the maximum energy is $\sqrt{s}=2.8 \mathrm{GeV}$ in comparison with $3.5 \mathrm{GeV}$ in Figs. 3 and 4. It is apparent from Figs. 6, 7 and 8 that the data are scarce 


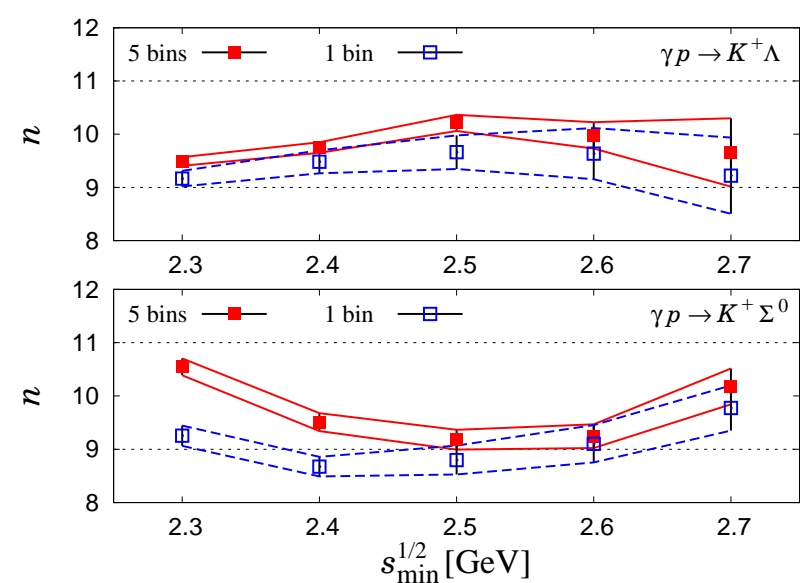

FIG. 5: (color online) Behavior of the scaling factor $n$ by changing the minimal value of the energy $\sqrt{s_{\min }}$ for fitting.

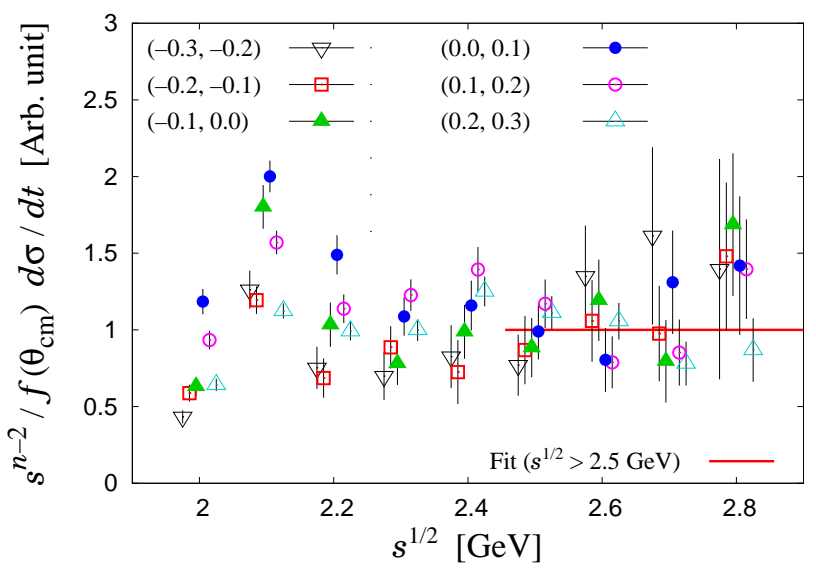

FIG. 6: (color online) Experimental data of $\gamma p \rightarrow K^{+} \Lambda(1405)$ cross section $d \sigma / d t$ [24] multiplied by $s^{n-2} / f\left(\theta_{\mathrm{cm}}\right)(n=10.6)$ with the function $f\left(\theta_{\mathrm{cm}}\right)$ in Eq. 6. The scaling factor $n$ is fixed at 10.6 by fitting in the region $\sqrt{s} \geq 2.5 \mathrm{GeV}$ as shown in Table $\Pi$ The solid line is a fit to the data. For a better visualization, we slightly shift the data to horizontal direction.

and the scaling property is less clear. Nevertheless, we tried to fit the cross section at high energies by the scaling behavior.

Fitted factors $n$ are shown in Table [1 for six-bin data as well as two-bin data and with various $\sqrt{s_{\min }}$ values from 2.3 $\mathrm{GeV}$ to $2.7 \mathrm{GeV}$. The results are shown in Fig. 9 by taking $\sqrt{s_{\min }}$ as the abscissa. The factors $n$ have large errors especially if the data are limited in the high-energy region, namely $\sqrt{s} \geq \sqrt{s_{\min }}=2.5,2.6$, or $2.7 \mathrm{GeV}$. For example, the factors $n$ for $\Lambda(1405)$ in Fig. 9 indicate that $\Lambda(1405)$ looks like a fivequark state $(n=11)$ if the data are used from $\sqrt{s_{\min }}=2.3$ or $2.4 \mathrm{GeV}$, whereas the factor becomes smaller $n<11$ with $\sqrt{s_{\min }} \geq 2.5 \mathrm{GeV}$. For $\Sigma(1385)^{0}$, the scaling factor is close to $n=11$ if the data with $\sqrt{s_{\min }}=2.3-2.5 \mathrm{GeV}$ are used; however, it becomes uncertain if only the data at higher energies, $\sqrt{s_{\min }}=2.6$ and $2.7 \mathrm{GeV}$, are analyzed. In terms of scaling behavior at $\sqrt{s_{\min }} \geq 2.5 \mathrm{GeV}, \Lambda(1405)$ and $\Lambda(1520)$ seem to be more consistent with three-quark system while

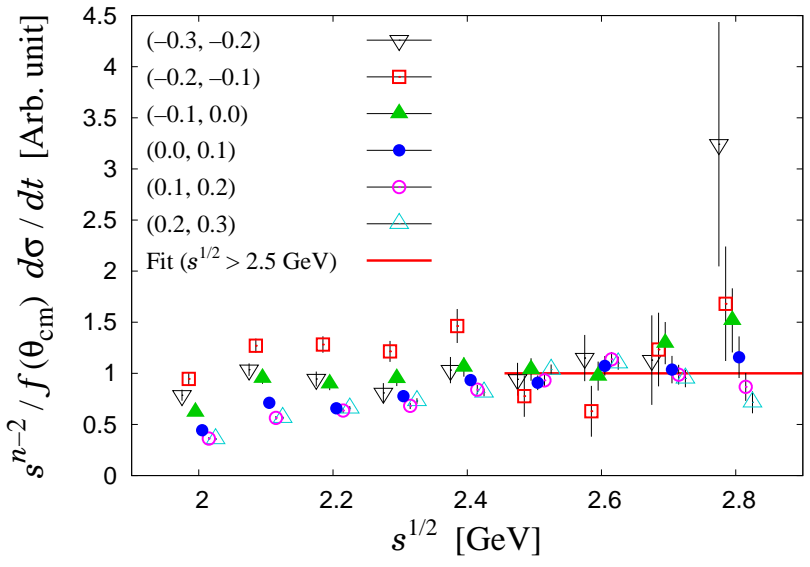

FIG. 7: (color online) Experimental data of $\gamma p \rightarrow K^{+} \Sigma(1385)^{0}$ cross section $d \sigma / d t$ [24] multiplied by $s^{n-2} / f\left(\theta_{\mathrm{cm}}\right)(n=11.4)$ with the function $f\left(\theta_{\mathrm{cm}}\right)$ in Eq. 6. The scaling factor $n$ is fixed at 11.4 by fitting in the region $\sqrt{s} \geq 2.5 \mathrm{GeV}$ as shown in Table $\Pi$ The solid line is a fit to the data. For a better visualization, we slightly shift the data to horizontal direction.

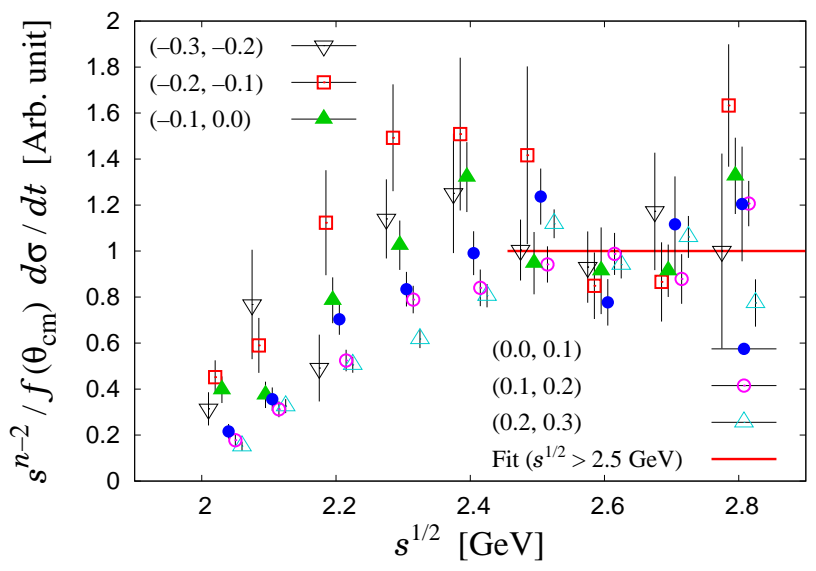

FIG. 8: (color online) Experimental data of $\gamma p \rightarrow K^{+} \Lambda(1520)$ cross section $d \sigma / d t$ [24] multiplied by $s^{n-2} / f\left(\theta_{\mathrm{cm}}\right)(n=9.8)$ with the function $f\left(\theta_{\mathrm{cm}}\right)$ in Eq. (6). The scaling factor $n$ is fixed at 9.8 by fitting in the region $\sqrt{s} \geq 2.5 \mathrm{GeV}$ as shown in Table $\Pi$ The solid line is a fit to the data. For a better visualization, we slightly shift the data to horizontal direction.

$\Sigma(1385)^{0}$ is close to five-quark one. Suppose a baryon is a mixture of conventional three-quark system, compact fivequark one, and diffuse meson-baryon molecule. Then, a transition in the scaling behavior at different energy region might be expected and the production at very high energies could be dominated by the state of minimal $n$, i.e., the relatively tight three-quark component $(n=9)$. Such a scenario seems favored by the observed $\sqrt{s_{\min }}$ dependence of scaling behavior for $\Lambda(1405)$. However, conclusive information of the scaling factors $n$ for the photoproductions of $\Lambda(1405), \Sigma(1385)^{0}$, and $\Lambda(1520)$ cannot be obtained from the current data, as seen from the scattered data even at high energies in Figs. 6, 7, and 8. 
TABLE II: Fitted values of the scaling factor $n$ for $\gamma p \rightarrow$ $K^{+} \Lambda(1405), K^{+} \Sigma(1385)^{0}$, and $K^{+} \Lambda(1520)$. We also show the $\chi^{2}$ values divided by the degrees of freedom $N_{\text {d.o.f. }}$.

\begin{tabular}{|c|c|c|}
\hline \multicolumn{3}{|c|}{$\gamma p \rightarrow K^{+} \Lambda(1405)$} \\
\hline$\sqrt{s_{\min }}$ & $n\left(\chi^{2} / N_{\text {d.o.f. }}\right), 6$ bins & $n\left(\chi^{2} / N_{\text {d.o.f. }}\right), 2$ bins \\
\hline $2.3 \mathrm{GeV}$ & $10.7 \pm 0.3 \quad(36 / 29)$ & $10.2 \pm 0.5 \quad(8 / 9)$ \\
\hline $2.4 \mathrm{GeV}$ & $11.3 \pm 0.4$ & $10.3 \pm 0.7$ \\
\hline $2.5 \mathrm{GeV}$ & $10.6 \pm 0.6$ & $9.0 \pm 1.1$ \\
\hline $2.6 \mathrm{GeV}$ & $9.6 \pm 1.0$ & $7.6 \pm 2.2$ \\
\hline $2.7 \mathrm{GeV}$ & $6.5 \pm 1.7$ & $5.2 \pm 4.7$ \\
\hline
\end{tabular}

\begin{tabular}{lllll}
\multicolumn{4}{c}{$\gamma p \rightarrow K^{+} \Sigma(1385)^{0}$} \\
$\sqrt{s_{\min }}$ & $n\left(\chi^{2} / N_{\text {d.o.f. }}\right), 6$ bins & $n\left(\chi^{2} / N_{\text {d.o.f. }}\right), 2$ bins \\
\hline $2.3 \mathrm{GeV}$ & $10.4 \pm 0.2$ & $(74 / 29)$ & $10.5 \pm 0.2$ & $(5 / 9)$ \\
$2.4 \mathrm{GeV}$ & $10.9 \pm 0.3$ & $(52 / 23)$ & $10.7 \pm 0.3$ & $(4 / 7)$ \\
$2.5 \mathrm{GeV}$ & $11.4 \pm 0.4$ & $(34 / 17)$ & $10.2 \pm 0.4$ & $(2 / 5)$ \\
$2.6 \mathrm{GeV}$ & $12.5 \pm 0.7$ & $(20 / 11)$ & $10.1 \pm 0.9$ & $(2 / 3)$ \\
$2.7 \mathrm{GeV}$ & $12.2 \pm 1.6$ & $(7 / 5)$ & $9.6 \pm 0.3$ & $(0 / 1)$
\end{tabular}

\begin{tabular}{|c|c|c|}
\hline & \multicolumn{2}{|c|}{$\gamma p \rightarrow K^{+} \Lambda(1520)$} \\
\hline$\sqrt{s_{\min }}$ & $n\left(\chi^{2} / N_{\text {d.o.f. }}\right), 6$ bins & $n\left(\chi^{2} / N_{\text {d.o.f. }}\right), 2$ bins \\
\hline $2.3 \mathrm{GeV}$ & $9.0 \pm 0.2 \quad(87 / 29)$ & $9.6 \pm 0.4 \quad(21 / 9)$ \\
\hline $2.4 \mathrm{GeV}$ & $9.4 \pm 0.3$ & $10.0 \pm 0.7$ \\
\hline $2.5 \mathrm{GeV}$ & $9.8 \pm 0.5$ & $9.3 \pm 1.1$ \\
\hline $2.6 \mathrm{GeV}$ & $8.7 \pm 0.7$ & $6.6 \pm 0.8$ \\
\hline $2.7 \mathrm{GeV}$ & $7.7 \pm 2.1$ & $5.8 \pm 1.9$ \\
\hline
\end{tabular}

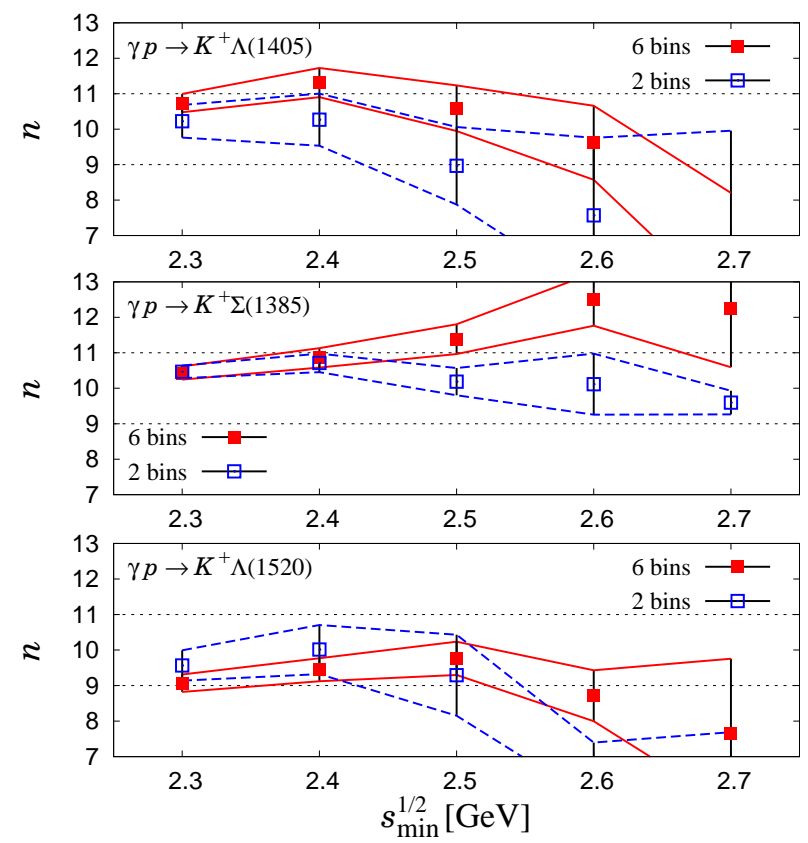

FIG. 9: (color online) Behavior of the scaling factor $n$ by changing the minimal value of the energy $\sqrt{s_{\min }}$ for fitting.

From our analysis, we propose that experimentalists measure the high-energy region $(\sqrt{s}>2.8 \mathrm{GeV})$ of the photoproduction cross sections to draw a solid conclusion for the scaling factor $n$. It should be especially important for an exotic hadron candidate, $\Lambda(1405)$. This kind of experiments should be possible at JLab after the $12-\mathrm{GeV}$ upgrade of its accelerator [31]. Similar measurements could be also done at LEPS (Laser Electron Photon beamline at SPring-8) II, especially because there is a plan to have a detector for large-angle scattering measurements [32] with the increase of photon energy. These high-energy experiments are important as a new direction of exotic hadron studies, which have been done so far in low-energy global observables such as spins, parities, masses, and decay widths.

\section{SUMMARY}

We analyzed the exclusive photoproduction data of hyperons, $\gamma p \rightarrow K^{+} Y\left[Y=\Lambda, \Sigma^{0}, \Lambda(1405), \Sigma(1385)^{0}\right.$, or $\Lambda(1520)$ ] by using the constituent-counting rule at high energies for determining the number of constituents involved in the exclusive reactions. Our studies intend to clarify the internal constituents of an exotic hadron candidate $\Lambda(1405)$, because the counting rule is considered to be valid for hadrons and nuclei, such as the proton, the pion, and deuteron. Therefore, our work can shed light on a new method of exotichadron studies for clarifying their internal structure by highenergy reactions where quark and gluon degrees of freedom are valid.

Our analysis results indicate that the data for the reactions $\gamma p \rightarrow K^{+} \Lambda$ and $\gamma p \rightarrow K^{+} \Sigma^{0}$ are consistent with the factor $n=9$. It suggests that $\Lambda$ and $\Sigma^{0}$ have three quark constituents in them, which is consistent with conventional quark models. On the other hand, at this moment it is difficult to pin down the factor $n$ for the photoproductions of $\Lambda(1405), \Sigma(1385)^{0}$, and $\Lambda(1520)$ due to lack of data. Nevertheless, the observed scaling behavior of hyperon $\Lambda(1405)$ seems to suggest that it is not purely three-quark or five-quark system. The number of constituent quarks for the excited hyperons could be determined by their photoproduction processes in principle if enough data are taken in a wide energy region. If an excited hyperon is a mixture of three-quark and five-quark states, energy dependence of the scaling behavior could provide valuable information for its composition and mixture.

The current method was not applied to exotic hadrons until recently, and it should be a new approach for identifying exotic hadrons and for clarifying their internal structure. The scaling for exotic hadron candidates should be clarified by precise cross-section measurements in future for the photoproductions in the high-energy region $(\sqrt{s}>2.8 \mathrm{GeV})$ and also hadronic exclusive reactions, for example, at J-PARC (Japan Proton Accelerator Research Complex) [33].

\section{Acknowledgments}

This work is partly supported by Grants-in-Aid for Scientific Research from MEXT and JSPS (No. 15K17649, No. 15J06538, and No. 25105010). 
[1] K. A. Olive et al. [Particle Data Group Collaboration], Chin. Phys. C 38, 090001 (2014).

[2] For a recent review on $\Lambda(1405)$, see T. Hyodo and D. Jido, Prog. Part. Nucl. Phys. 67, 55 (2012). For lattice QCD studies, see J. M. M. Hall et al., Phys. Rev. Lett. 114, 132002 (2015).

[3] A. Bondar et al. [Belle Collaboration], Phys. Rev. Lett. 108, 122001 (2012).

[4] R. Aaij et al. [LHCb Collaboration], Phys. Rev. Lett. 115, 072001 (2015).

[5] S. J. Brodsky and G. R. Farrar, Phys. Rev. Lett. 31, 1153 (1973).

[6] S. J. Brodsky and G. R. Farrar, Phys. Rev. D 11, 1309 (1975).

[7] R. L. Anderson, D. Gustavson, D. Ritson, G. A. Weitsch, H. J. Halpern, R. Prepost, D. H. Tompkins, and D. E. Wiser, Phys. Rev. D 14, 679 (1976).

[8] L. Y. Zhu et al. [Jefferson Lab Hall A Collaboration], Phys. Rev. Lett. 91, 022003 (2003).

[9] L. Y. Zhu et al. [Jefferson Lab Hall A and Jefferson Lab E94104 Collaborations], Phys. Rev. C 71, 044603 (2005).

[10] R. A. Schumacher and M. M. Sargsian, Phys. Rev. C 83, 025207 (2011).

[11] B. Dey, Phys. Rev. D 90, 014013 (2014).

[12] B. R. Baller et al., Phys. Rev. Lett. 60, 1118 (1988); C. White et al., Phys. Rev. D 49, 58 (1994).

[13] Y. N. Uzikov, JETP Lett. 81, 303 (2005).

[14] Y. Ilieva, Few Body Syst. 54, 989 (2013).

[15] H. Kawamura, S. Kumano, and T. Sekihara, Phys. Rev. D 88, 034010 (2013).

[16] H. Kawamura and S. Kumano, Phys. Rev. D 89, 054007 (2014).

[17] M. V. Galynskii and E. A. Kuraev, Phys. Rev. D 89, 054005
(2014).

[18] S. H. Blitz and R. F. Lebed, Phys. Rev. D 91, 094025 (2015).

[19] S. J. Brodsky and R. F. Lebed, Phys. Rev. D 91, 114025 (2015).

[20] T. Hyodo, D. Jido and A. Hosaka, Phys. Rev. C 85, 015201 (2012).

[21] T. Hyodo, Int. J. Mod. Phys. A 28, 1330045 (2013).

[22] T. Sekihara and S. Kumano, Phys. Rev. C 89, 025202 (2014).

[23] T. Sekihara, T. Hyodo and D. Jido, PTEP 2015, 063 D04 (2015) arXiv:1411.2308 [hep-ph]].

[24] K. Moriya et al. [CLAS Collaboration], Phys. Rev. C 88, 045201 (2013).

[25] F. Aceti, E. Oset and L. Roca, Phys. Rev. C 90, 025208 (2014).

[26] M. E. McCracken et al. [CLAS Collaboration], Phys. Rev. C 81, 025201 (2010).

[27] B. Dey et al. [CLAS Collaboration], Phys. Rev. C 82, 025202 (2010).

[28] A. H. Mueller, Phys. Rep. 73, 237 (1981); S. J. Brodsky and G. P. Lepage, Adv. Ser. Direct. High Energy Phys. 5, 93 (1989).

[29] B. Aubert et al. [BaBar Collaboration], Phys. Rev. D 80, 052002 (2009); S. Uehara et al. [Belle Collaboration], Phys. Rev. D 86, 092007 (2012).

[30] S. A. Pereira et al. [CLAS Collaboration], Phys. Lett. B 688, 289 (2010).

[31] J. Dudek et al., Eur. Phys. J. A 48, 187 (2012).

[32] M. Niiyama, EPJ Web of Conferences, 37, 01006 (2012).

[33] W.-C. Chang, S. Sawada, T. Sawada, J.-C. Peng et al., in preparation for a proposal to J-PARC. 